Research Article

Genetics of Microorganisms

\title{
The PAC-3 transcription factor critically regulates phenotype-associated genes in Neurospora crassa
}

\author{
Maíra Pompeu Martins ${ }^{1}$ (D), Nilce Maria Martinez-Rossi ${ }^{1}$ (D), Pablo Rodrigo Sanches ${ }^{1}$ (D) and Antonio \\ Rossi $^{1}$ \\ ${ }^{1}$ Universidade de São Paulo, Faculdade de Medicina de Ribeirão Preto, Departamento de Genética, \\ Ribeirão Preto, SP, Brazil
}

\begin{abstract}
Transcription factors play an important role in fungal environmental adaptive process by promoting adjustment to challenging stimuli via gene modulation and activation of signaling networks. The transcription factor encoded by the pac-3/rim101/pacC gene is involved in $\mathrm{pH}$ regulation and is associated with a wide variety of cellular functions. The deletion of pac-3 affects fungal development. In Neurospora crassa, the $\Delta$ pac-3 strain presents diminished aerial growth and reduced conidiation. However, the PAC-3-regulated genes associated with this altered phenotype have not been elucidated. In this study, we used RNA-seq to analyze the phenotypic plasticity induced after pac-3 deletion in the filamentous fungus $N$. crassa cultivated in media supplemented with sufficient or limited inorganic phosphate. Genes related to morphology, hyphal development, and conidiation were of particular interest in this study. Our results suggest a pac-3 dependency in gene regulation in a Pi-dependent manner. Furthermore, our analysis suggested that the fungus attempts to overcome the deletion effects in a $\Delta$ pac-3 mutant through a complex combined regulatory mechanism. Finally, the modulatory responses observed in the $\Delta$ pac-3 strain, a double mutant generated based on the $\Delta m u s-52$ mutant strain, is strain-specific, highlighting that the phenotypic impact may be attributed to pac-3 absence despite the combined mus-52 deletion.
\end{abstract}

Keywords: RNA-seq, conidiation, hyphal development, transcription factor, inorganic phosphate.

Received: November 06, 2019; Accepted: May 08, 2020.

\section{Introduction}

To survive and proliferate, fungi must interact with and sense changes in their environment (Trevisan et al., 2011). The adaptive success in different niches is resultant of the ability to scavenge for nutrients and respond to several challenging factors such as extreme temperature, carbon source, and pH changes (Han et al., 1987; Bahn et al., 2007; Martinez-Rossi et al., 2017). Fungal sensing of the environment leads to the activation of intracellular signaling pathways, which are mediated by transcription factors. This network results in stress-associated transcriptional patterns which support the adaptation to specific stimuli (Braunsdorf et al., 2016; Simaan et al., 2019). The $\mathrm{pH}$ fitness of fungi is directly mediated by a $\mathrm{C} 2 \mathrm{H} 2$ zinc finger transcription factor encoded by the pac-3/rim 101/pacC gene, which is activated through the highly conserved Pal/Rim signaling pathway (Trevisan et al., 2011; Rossi et al., 2013). The $\mathrm{pH}$ signaling cascade activates gene regulation in response to acidic to alkaline $\mathrm{pH}$ shifts, which can extensively alter metabolic events (Rossi et al., 2013).

Send correspondence to Nilce Maria Martinez-Rossi. Universidade de São Paulo, Faculdade de Medicina de Ribeirão Preto, Departamento de Genética, Av. Bandeirantes, 3900, 14049-900, Ribeirão Preto, SP, Brazil. E-mail: nmmrossi@usp.br.
Using mutant strains carrying the $\Delta p a c-3$ (or rim 101/pacC) revealed that the PAC-3 transcription factor correlates with fungal traits beyond $\mathrm{pH}$ signaling, impacting fundamental biological processes, including cell morphology, hyphae growth, conidiation, and adaptation to the host or nutritional variances (Ferreira-Nozawa et al., 2006; Mendes et al., 2012; O'Meara et al., 2014; Martins et al., 2018, 2019; Rascle et al., 2018). In Neurospora crassa, the deletion of pac-3 and the pal cascade-associated genes, except for $\Delta$ pal-9/palI, diminished aerial growth, reduced conidiation, and resulted in high production of melanin, in comparison with that in the wild-type strain (Virgilio et al., 2016). A decrease in conidiation was also observed in the Trichophyton interdigitale H6 pacC mutant (FerreiraNozawa et al., 2006). Additionally, the absence of pacC led to low conidiation in Botrytis cinerea (Rascle et al., 2018), Magnaporthe oryzae (Landraud et al., 2013), and Aspergillus nidulans (Tilburn et al., 1995). Thus, there is a strong connection between the PAC-3 transcription factor and fungal development. However, the regulatory effect resultant of PAC-3 deletion on genes responsible for fungal development remains unexplored.

The expression of $\mathrm{pac}-3 / \mathrm{rim} 101 / \mathrm{pacC}$ is modulated in response to environmental conditions, including variations in inorganic phosphate (Pi), carbon sources, and $\mathrm{pH}$ fluctua- 
tions, which may impair critical physiological functions (Ferreira-Nozawa et al., 2006; Trevisan et al., 2011; Mendes et al., 2012; Martins et al., 2019). In this study, we used RNA-seq analysis to assess the genes related to morphogenesis and development of the $N$. crassa mutant $\Delta p a c-3$ cultivated in media containing sufficient or limited $\mathrm{Pi}$, an essential constituent of biomolecules (Gras et al., 2013). The purpose was to obtain evidence for transcriptional contribution to the observed phenotypical patterns. Pi is involved in diverse metabolic pathways and thus functions as a growthlimiting factor in microorganisms (Dick et al., 2011; Vicent et al., 2015). To determine whether pac-3-regulated genes are involved in the $N$. crassa phenotype, we studied the transcriptional impact that resulted from both the deletion and nutrient adaptive response simultaneously, as well as the changes that occurred exclusively due to the gene deletion independently of the nutritional condition. The results obtained provide evidence of the role of pac-3-mediated regulation in N. crassa growth and development, and indicate the genes possibly associated with the phenotypical effects observed in the $\Delta p a c-3$ mutant.

\section{Material and Methods}

\section{Culture conditions of $N$. crassa knockout strains}

N. crassa mus-52 $2^{\mathrm{KO}}$ (FGSC\#9568) parental and $\Delta p a c$ 3 (NCU00090) knockout strains (Cupertino et al., 2012) were maintained on solid Vogel's Minimal (VM) medium, pH 5.8 (Vogel, 1956) containing $2 \%$ sucrose at $30{ }^{\circ} \mathrm{C}$. Approximately $10^{7}$ cells $/ \mathrm{mL}^{-1}$ conidia were germinated in an orbital shaker for $5 \mathrm{~h}$ at $30{ }^{\circ} \mathrm{C}(200 \mathrm{rpm})$, as previously described (Martins et al., 2019), in low- and high-Pi media (final concentrations, $10 \mu \mathrm{M}$ or $10 \mathrm{mM} \mathrm{Pi}$, respectively). The media was supplemented with $44 \mathrm{mM}$ sucrose as the carbon source and adjusted to $\mathrm{pH} 5.4$ with $50 \mathrm{mM}$ sodium citrate (Nyc et al., 1966; Gras et al., 2007). The resulting mycelia was collected by filtering through $0.22-\mu \mathrm{m}$ size filters (Millipore Corp., USA), frozen in liquid nitrogen, and stored at $80^{\circ} \mathrm{C}$ until RNA extraction. Experiments were performed in three biological replicates.

\section{RNA extraction, sequencing, data analysis, and functional enrichment}

Total RNA was isolated using TRIzol Reagent (Invitrogen, USA) according to the manufacturer's instructions and treated with DNase I, RNase-free (Thermo Fisher, USA). The RNA concentration was quantified using a NanoDrop ND-1000 spectrophotometer (Thermo Fisher). The RNA integrity was determined by agarose formaldehyde gel electrophoresis and using the Agilent Bioanalyzer platform 2100 (Agilent, USA). Purity and concentration were measured using a NanoDrop ND-1000 spectrophotometer (Thermo Fisher). A total of 12 libraries ( $\Delta m u s-52$ and $\Delta p a c$ - 3 strains, cultivated in high- and low-Pi concentration media, each, in three biological replicates) were sequenced on an Illumina HiSeq2000 (Illumina, USA) se- quencer platform with paired-end 100-bp reads. RNA-seq data were analyzed and validated as previously described (Martins et al., 2019) and deposited at the GEO database with accession number GSE132373.

\section{Selection of morphology- and development-related genes}

After functional annotation analysis with the Blast2GO tool, the genes identified in the RNA-seq data were filtered with a customized $\mathrm{R}$ script, mapping modulated Genes and Gene Ontology (GO) terms. The script detected the descendant terms of the Gene Ontology nodes "cell wall," "developmental process," "cellular developmental process," "regulation of biological process," and "anatomical structure development" with the Bioconductor GO.db package (Carlson, 2018). We further identified morphology and development-related genes using literature data. The identified genes are listed in Table 1.

\section{In silico evaluation of the putative PAC-3-binding sites}

The $N$. crassa OR74A genome (Ensembl Fungi) was used to search for the occurrence of the PAC-3 motif, 5'GCCARG-3' (Tilburn et al., 1995), in the 5' upstream regions (1 kb) of each gene (Table S1). The pursuit was determined using an ad hoc Perl script (Martins et al., 2019).

\section{Results}

\section{Global DEG identification and selection of genes associated with morphogenesis and development in the RNA-seq libraries}

To perform a comprehensive analysis of the impact of $\Delta p a c-3$ knockout on gene modulation, and to determine the effects of Pi variation on that response, we evaluated the results obtained by high-throughput sequencing (RNA-seq) using the $N$. crassa mus $-52^{\mathrm{KO}}$ background strain as the control. By applying a cut-off threshold of at least 2.8-fold difference and a statistical significance threshold of $\mathrm{P}<0.05,427$ genes were identified as differentially modulated in response to both of the analyzed Pi conditions (Martins et al., 2019). The results identified 55 genes that are associated with morphogenesis and development (Table 1). A heatmap of gene expression of the identified genes is shown in Figure 1. MultiExperiment Viewer (MeV) was used for hierarchical clustering by average linkage clustering based on Pearson correlation.

\section{Gene modulation in response to pac-3 deletion}

Among the identified genes that associated with the $N$. crassa developmental progress, catalase-3 (NCU00355), ornithine-N5-oxygenase (NCU07117), 1,3-beta-glucanosyltransferase gell (NCU07253), two hypothetical proteins (NCU08351 and NCU01064), and two $\mathrm{Ca}^{2+}$-ATPases (E1E2 ATPase-1, NCU05046; and calcium-transporting ATPase3, NCU07966) were repressed in both Pi conditions. Among the genes that were upregulated in response to pac-3 
Table 1 - Genes of $N$. crassa modulated in response to low-Pi or high-Pi concentrations. Comparisons were made for the double mutant $\Delta p a c-3$ strain considering the $\Delta m u s-52$ strain as control (Martins et al., 2019) and for $\Delta m u s-52$ strain considering the St.L.74-OR23-1VA strain as control (Martins et al., 2018). The selected genes are associated with morphology and development regulation, including the Gene Ontology descendants search of the nodes "cell wall," "developmental process," "cellular developmental process," "regulation of biological process," and "anatomical structure development".

\begin{tabular}{|c|c|c|c|c|c|c|}
\hline \multirow[t]{2}{*}{ GO ID } & \multirow[t]{2}{*}{ Gene ID } & \multirow[t]{2}{*}{ Gene Product Name } & \multicolumn{2}{|c|}{$\Delta p a c-3$ vs. $\Delta m u s-52$} & \multicolumn{2}{|c|}{$\Delta m u s-52$ vs. $74 \mathrm{~A}$} \\
\hline & & & low-Pi & high-Pi & low-Pi & high-Pi \\
\hline GO:0006355 & NCU04058 & BZIP domain-containing protein & & 3.00 & 2.77 & \\
\hline $\begin{array}{l}\text { GO:0043666 } \\
\text { GO:0070884 }\end{array}$ & NCU01504 & Calcineurin binding protein & & 2.15 & & \\
\hline GO:0006357 & NCU08852 & Poly(ADP-ribose) polymerase & & 2.18 & 2.66 & \\
\hline $\begin{array}{l}\text { GO:0030435 } \\
\text { GO:0048315 } \\
\text { GO:0006357 }\end{array}$ & NCU08726 & Fluffy & & 1.94 & & \\
\hline GO:0006355 & NCU03650 & DNA repair protein RAD16 & & 1.55 & 1.77 & \\
\hline Unannotated & NCU04197 & CipC protein & 6.62 & 8.44 & & \\
\hline GO:0006357 & NCU00282 & $\mathrm{Zn}(2)$-C6 fungal-type domain-containing protein & 3.89 & 4.34 & & \\
\hline GO:0046830 & NCU07723 & Norsolorinic acid reductase & 3.08 & 2.26 & & \\
\hline GO:0006357 & NCU04866 & All development altered- 6 & 2.00 & 2.15 & & \\
\hline GO:0033499 & NCU04442 & GAL10 & 1.73 & 1.77 & & \\
\hline $\begin{array}{l}\text { GO:0000122 } \\
\text { GO:0001080 } \\
\text { GO:0045944 } \\
\text { GO: } 1903833\end{array}$ & NCU08055 & B-ZIP transcription factor IDI4 & 1.60 & 2.45 & & \\
\hline GO:0007165 & NCU06111 & GTPase Ras2p & 1.55 & 1.52 & & \\
\hline Unannotated & NCU09629 & Hypothetical protein & -1.81 & 2.12 & & -3.14 \\
\hline Unannotated & NCU04605 & Hypothetical protein & -2.61 & & & -3.39 \\
\hline GO:0043934 & NCU00586 & Non-anchored cell wall protein- 6 & -2.48 & & & \\
\hline GO:0006355 & NCU00090 & PH-response transcription factor pacC/RIM101 & -1.80 & & & \\
\hline GO:0045013 & NCU03965 & Catabolite repression protein creC & -1.78 & & & \\
\hline GO:0072344 & NCU03367 & Hypothetical protein & -1.78 & & & \\
\hline $\begin{array}{l}\text { GO:0005619 } \\
\text { GO:0048315 }\end{array}$ & NCU08791 & Catalase-1 & -1.64 & & & -2.12 \\
\hline GO:0061414 & NCU02142 & $\mathrm{Zn}(2)$-C6 fungal-type domain-containing protein & -1.55 & & -4.62 & -4.27 \\
\hline GO:0030968 & NCU02235 & Glycosyl hydrolase family $47-6$ & & -6.03 & & \\
\hline $\begin{array}{l}\text { GO:0006355 } \\
\text { GO:0006357 }\end{array}$ & NCU01931 & Hypothetical protein & & -1.98 & & \\
\hline GO:0030435 & NCU00399* & Cell wall protein PhiA & & -1.79 & & \\
\hline GO:0007264 & NCU02167 & Krev-1-like & & -1.57 & & \\
\hline Unannotated & NCU01064 & Hypothetical protein & -8.84 & -5.77 & & \\
\hline GO: 1902600 & NCU05046 & E1-E2 ATPase-1 & -6.77 & -5.72 & & \\
\hline GO:0055114 & NCU04452 & Menadione induced gene-3 & -5.18 & -5.77 & & \\
\hline GO: 1902600 & NCU07966 & Calcium-transporting ATPase 3 & -4.00 & -3.88 & & \\
\hline GO:0009277 & NCU07253* & 1,3-beta-glucanosyltransferase gel1 & -3.56 & -3.26 & & \\
\hline GO:0016787 & NCU07117 & Ornithine-N5-oxygenase & -3.02 & -2.28 & & \\
\hline GO:0061414 & NCU00155 & C6 transcription factor & -2.10 & -1.86 & & \\
\hline
\end{tabular}

Gene expression levels represented as $\log _{2}$-fold change comparing test and control strains in each of the Pi conditions ( $\Delta$ pac-3 vs. $\Delta m u s-52$ or $\Delta m u s-52 v s$. N. crassa St.L.74-OR23-1VA, identified as 74A strain in the table). Only values of $\log 2$ FoldChange $>=1.5$ or $<=-1.5$ were represented.

$\$$ Genes presenting binding motif for PAC-3 in their promoter region.

${ }^{\dagger}$ Transcription factors

deletion, regardless of the Pi condition, we identified CipC protein (NCU04197), GAL10 (NCU04442), GTPase Ras2p (NCU06111), and the anchored cell wall protein-12 (NCU08171).

\section{Gene modulation in response to Pi variation}

The cell wall protein PhiA (NCU00399), fluffy (NCU08726), three hypothetical proteins identified as
NCU09629, NCU04493, and NCU04605, a non-anchored cell wall protein-6 (NCU00586), and the galactose oxidase (NCU09209) were identified among the Pi-dependent genes.

\section{Discussion}

Deciphering the regulatory mechanisms underlying the developmental programs in fungi can contribute to the elucidation of events that are unique and essential to these 


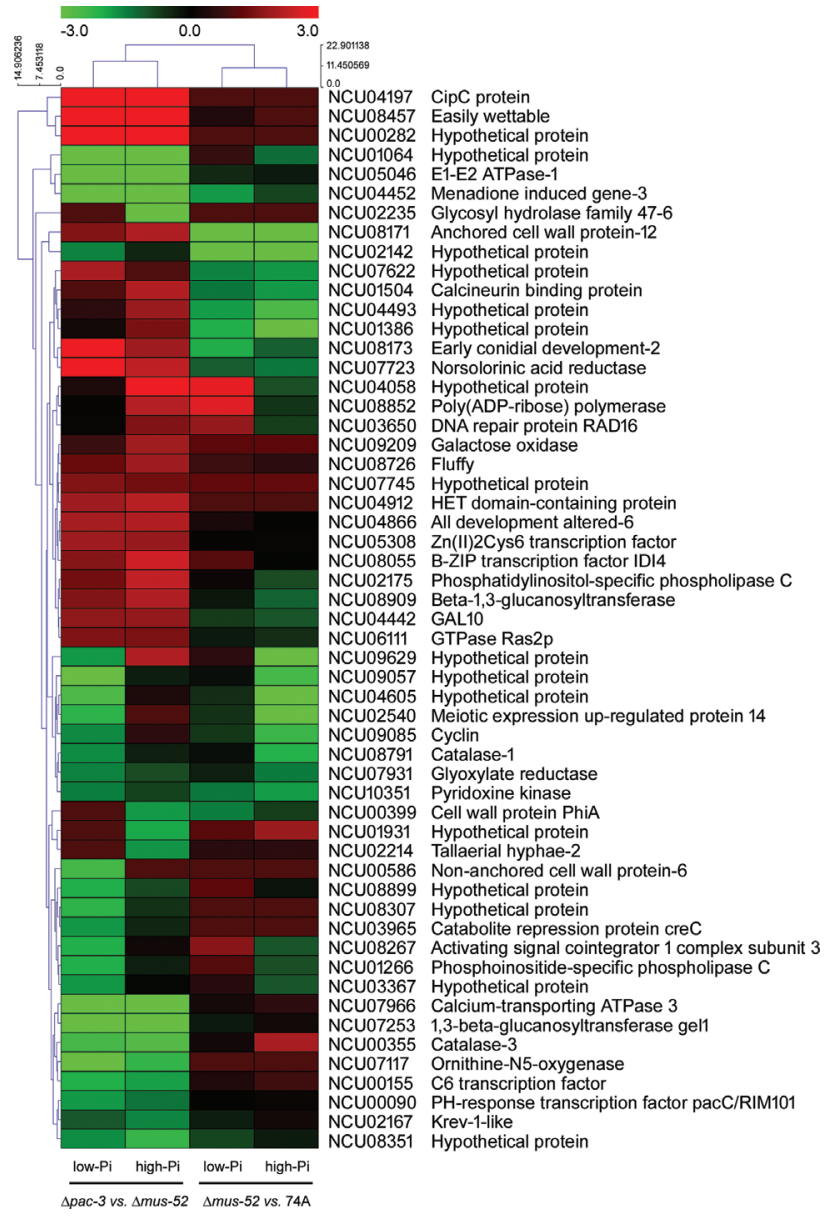

Figure 1 - Heatmap of morphology and development regulation-associated genes from $N$. crassa mutants. The hierarchical clustering of expression patterns for the 55 differentially expressed genes identified in the $\Delta p a c-3 \Delta m u s-52$ mutant strain versus $\Delta m u s-52$ is compared to the modulatory pattern observed for the same genes in the $\Delta m u s-52$, evaluated in comparison to the wild-type strain. Expression levels were loaded into the MultiExperiment Viewer (MeV) and analyzed using average-linkage hierarchical clustering with a Pearson correlation coefficient distance metric. The intensity of color represents the value of upregulation (red) or downregulation (green) in $\log 2 \mathrm{FC}$.

microorganisms. In this study, we aimed to identify the pac-3-regulated genes involved in N. crassa development in a transcriptional scenario, and thus contribute to the molecular understanding of the phenotype observed in the $\Delta p a c-3$ mutant. Reduced growth and low conidiation were not observed in the parental background strain $m u s-52^{\mathrm{KO}}$, which indicated that the fungal development defects were due to the deletion of pac-3. Although pac-3 deletion drastically affected the $N$. crassa phenotype, only a small number of genes associated with cell morphology, or hyphal and conidial development, were modulated in the evaluated conditions of Pi variance (Table 1). The phenotypic effect in the $\Delta p a c-3$ strain may be the result of a more complex combined regulatory mechanism. Among the 55 genes that were identified, 31 possessed a binding motif for PAC-3 in their promoter regions (Table $\mathrm{S} 1$ ). The deletion of pac-3 resulted in the differential modulation of 12 additional transcription factors, seven of which contained the PAC- 3 binding motif.

\section{$N$. crassa gene downregulation is reflected in its phenotype}

The transcriptional response that resulted explicitly from the absence of PAC-3, with Pi conditions being not a determinant of gene modulation, involved the downregulation of two catalase genes. Catalase-3 gene deletion provoked enhanced conidial production, hyphal adhesion, and more aerial hyphae in N. crassa (Michan et al., 2003; Sun et al., 2012), whereas catalase-1 deletion increased germination rate in Metarhizium anisopliae (Morales Hernandez et al., 2010). Since catalase activity is protective against cellular component damage, the gene repression observed in this study may have led to oxidative stress toxic effects, which would impair $N$. crassa development.

Our results also identified that the ornithine-N5-oxygenase gene is downregulated. The encoded enzyme catalyzes the first step in the microbial-exclusive hydroxamate siderophore biosynthesis system (Eisendle et al., 2003; Hissen et al., 2005). Disruption of orthologue genes in $\mathrm{A}$. nidulans and Nomuraea rileyi correlated the activity of the siderophore biosynthesis to decreased conidiation and defective hyphae elongation (Eisendle et al., 2003; Li et al., 2016).

Furthermore, 1,3-beta-glucanosyltransferase gell, homologous to gas 1 in Saccharomyces cerevisiae, and phrl in Candida albicans, is downregulated. This gene encodes a GPI-anchored protein, required for correct morphogenesis and polar growth in these organisms (Ragni et al., 2011). In S. cerevisiae, gas 1 deletion reduced fungi growth rate, and at an alkaline $\mathrm{pH}$, the apical growth in the $\Delta p h r l$ mutant was compromised in both yeast and hyphal growth forms (Mouyna et al., 2000).

Among the downregulated hypothetical proteins, the gene identified as NCU08351 is directly associated with reduced conidial production, based on its knockout mutant strain (Sun et al., 2012), and the gene identified as NCU01064 is related to the conidiation protein CON-6 (Suzuki et al., 2013). The latter is a conidiation-specific time-dependent activator and is associated with the induction of N. crassa development (Bailey and Ebbole, 1998).

Two calcium-transporting pump genes, which are implicated in the maintenance of the proper level of calcium within cells, were repressed. The E1-E2 ATPase-1 and the calcium-transporting ATPase 3 are associated with hyphal morphogenesis in N. crassa (Silverman-Gavrila and Lew, 2001; Zelter et al., 2004). In our results, morphology and development genes modulated in response to PAC-3 absence were highly repressed. The observed repression supported the observed reduced conidial production, in comparison with that in the wild-type strain. Further, the induction of other related genes suggested an attempt of the mutant fungi to overcome pac-3 deletion. 


\section{$\Delta$ pac-3-mutant attempts to restore developmental deregulation}

We identified that CipC (from concanamycin-induced protein) protein was highly upregulated in both Pi conditions. This protein, exclusively found in fungi (Asif et al., 2006), was associated with filamentous growth in Ustilago maydis (Rodriguez-Kessler et al., 2012), and with hyphal development and conidial surface interactions in the pathogenic mold Aspergillus fumigatus (Asif et al., 2006; Bauer et al., 2010). In A. nidulans, the upregulation of CipC protein in a mutant strain deficient in glucosidase I could be one of the factors that contributed to the hyperbranching, resultant of its activity in polarizing growth (Zhang et al., 2009).

The UDP-galactose-4-epimerase (GAL10), which was also upregulated, codes for an enzyme of the galactose metabolism. Its activity is associated with cell-wall integrity, morphology, and induces excessive filamentation in C. albicans (Singh et al., 2007), and results in highly branched hyphae and reduced conidiation in A. nidulans (El-Ganiny et al., 2010). We also identified the induction of the GTPase Ras $2 p$, which codes for GTPase signal transducer proteins and acts as a regulator of growth and development (Fortwendel, 2015). In N. crassa, this protein is reported to be involved in the regulation of cell morphology, which affects the apical growth of hyphae and conidium formation (Kana-uchi et al., 1997).

Proteomic analysis identified proteins that are secreted by the vegetative hyphae of $N$. crassa and expressed in a cell-type-specific manner (Maddi et al., 2009). One such protein, anchored cell wall protein-12, is implicated in cell wall remodeling and in the growth of the hyphae (Potapova, 2014). Our results revealed that the gene coding for this protein is induced in N. crassa in both Pi conditions. Our findings identified the overexpression of some developmental genes in the mutant strain, which indicates a possible attempt of the fungi to compensate for pac-3 deletion.

\section{$\Delta$ pac-3-mutation is responsive to $\mathrm{Pi}$ variation}

Among the genes presenting the pac-3 binding motif, upon induction, the up-modulation occurred in high-Pi as a pattern. When repressed, with four exceptions (NCU02235, NCU01931, NCU00399, and NCU02167), the downregulation occurred in low Pi. Thus, our results suggest a pac-3 dependency in gene regulation in a Pi-dependent manner.

The cell wall protein PhiA, an essential gene for conidia development and healthy growth in A. nidulans (Melin et al., 2003), is repressed exclusively in high Pi conditions, suggesting that the Pi restriction directly impacts gene modulation. The Fluffy gene is induced in response to sufficient $\mathrm{Pi}$. This gene codes for a C6 zinc finger transcription factor active in the regulation of two out of the five genes that act as specific regulators of conidiation in $N$. crassa (Mendes et al., 2016). Fluffy is expressed at a basal level in vegetative hyphae, and is transcriptionally activated in the aerial hyphae formation (Bailey and Ebbole, 1998; Rerngsamran et al., 2005; Mendes et al., 2016). The expression pattern of PhiA and Fluffy genes in high Pi suggests that $\Delta p a c-3$ strain shows an attempt to restore conidiation by activating genes other than PhiA in the herein evaluated conditions.

Nineteen conidia-specific cell wall proteins were identified using a proteomic approach with N. crassa (Ao et al., 2016). The promoter regions of each coding gene were analyzed to determine whether they were expressed in a conidiaspecific manner (Ao et al., 2016). Among these genes, four were modulated in our results. Two of them, identified as non-anchored cell wall protein-6 (NCU00586) and the hypothetical protein NCU04605 were downregulated in response to low-Pi. The other two, hypothetical protein NCU04493 and galactose oxidase (NCU09209), were induced in high-Pi. The two upregulated genes identified had conidiation-specific expression using the promoter approach, and the two downregulated genes, did not give a conidia-specific developmental expression pattern. These complementary results support the feasible role of the transcription factor pac-3 in conidia-related regulation in high Pi.

The hypothetical protein NCU09629 presented with inverted modulation in Pi conditions. In limited Pi availability, this gene is repressed, and under sufficient $\mathrm{Pi}$, it is induced. This gene shows the HET (for heterokaryon incompatibility [HI]) domain, a regulator of $\mathrm{HI}$, and is associated with severe growth inhibition and negatively affects conidiation and hyphal compartmentation that leads to programmed cell death (Dementhon et al., 2006).

\section{Genetic interactions render unexpected phenotype}

The strain under analysis is a double mutant ( $\triangle p a c-$ $3 \Delta m u s-52)$. As described for negative genetic interactions (Mani et al., 2008), the resultant phenotype was more substantial than expected. These genetic interactions frequently involve genes presenting leastwise partially overlapping functions, which may compensate for the deletion mutually (Hartman et al., 2001). Growth-based gene interaction profiling is reasonable to subdivide the observed negative to positive interactions, although expression-based genetic interaction profiling provides a more specific understanding of the genetic interaction patterns (Amini et al., 2019).

A heatmap that depicts the relative expression levels of the growth and development-associated genes identified in the $\Delta p a c-3 \Delta m u s-52$ mutant strain compared to the expression in the corresponding single mutant $\Delta m u s-52$ is shown in Figure 1. As a pattern, the modulatory response observed in the single mutant was not sustained in the double mutant strain. Two profiles could be highlighted: in one of them, the modulatory pattern was strain-specific, as observed in the induced cluster including the gene identified as $\mathrm{CipC}$ protein (NCU04197) and the hypothetical protein NCU00282, or in the repressed cluster including the calcium-transporting ATPase 3 (NCU07966) and Krev-1-like (NCU02167) genes. In the second explicit expression profiling, the pattern skipped between high to low Pi in the different strains, as observed in a cluster, including the hypothetical protein NCU09629 and catalase-1 (NCU08791). In only a few genes, the expression pattern was maintained in both conditions and strains as for the pyridoxine kinase (NCU10351). 
Our results provide evidence of the role of the double mutant-mediated regulation in $N$. crassa. In the same proportion that mus-52 deletion incurs underestimated consequences to the organism (Martins et al., 2018), the associated pac-3 deletion reflects a pervasive and profound effect in $N$. crassa development, bringing relevant insights regarding biological networks.

\section{Acknowledgments}

We acknowledge funding from the Research Support Foundation of São Paulo State - FAPESP (Grant No. 2014/03847-7, and Postdoctoral Fellowship No. 2018/11319-1); the National Council for Scientific and Technological Development - CNPq (Grants No. 305797/2017-4, and 304989/2017-7); Coordination for the Improvement of Higher Education Personnel - CAPES (Finance Code 001), and Foundation for Support to Teaching, Research and Assistance - FAEPA of the HCFMRP-USP. We thank C. A. Vieira and S. H. Castrechini for technical support, and Dr. Maria Celia Bertolini for kindly providing the knockout mutants.

\section{Conflict of Interest}

The authors declare no conflict of interest.

\section{Author Contributions}

MPM conducted the experiments, analyzed the data, and drafted the manuscript. PRS performed computational and statistical analyses. AR and NMM-R designed the project, supervised the research, and prepared the manuscript. All authors read and approved the final version.

\section{References}

Amini S, Jacobsen A, Ivanova O, Lijnzaad P, Heringa J, Holstege FCP, Feenstra KA and Kemmeren P (2019) The ability of transcription factors to differentially regulate gene expression is a crucial component of the mechanism underlying inversion, a frequently observed genetic interaction pattern. PLoS Comput Biol 15:e1007061.

Ao J, Aldabbous M, Notaro MJ, Lojacono M and Free SJ (2016) A proteomic and genetic analysis of the Neurospora crassa conidia cell wall proteins identifies two glycosyl hydrolases involved in cell wall remodeling. Fungal Genet Biol 94:47-53.

Asif AR, Oellerich M, Amstrong VW, Riemenschneider B, Monod $\mathrm{M}$ and Reichard U (2006) Proteome of conidial surface associated proteins of Aspergillus fumigatus reflecting potential vaccine candidates and allergens. J Proteome Res 5:954-962.

Bahn YS, Xue C, Idnurm A, Rutherford JC, Heitman J and Cardenas ME (2007) Sensing the environment: lessons from fungi. Nat Rev Microbiol 5:57-69.

Bailey LA and Ebbole DJ (1998) The fluffy gene of Neurospora crassa encodes a Gal4p-type C6 zinc cluster protein required for conidial development. Genetics 148:1813-1820.

Bauer B, Schwienbacher M, Broniszewska M, Israel L, Heesemann J and Ebel F (2010) Characterisation of the CipC-like protein AFUA_5G09330 of the opportunistic human pathogenic mould Aspergillus fumigatus. Mycoses 53:296-304.

Braunsdorf C, Mailander-Sanchez D and Schaller M (2016) Fungal sensing of host environment. Cell Microbiol 18:1188-1200.
Cupertino FB, Freitas FZ, de Paula RM and Bertolini MC (2012) Ambient $\mathrm{pH}$ controls glycogen levels by regulating glycogen synthase gene expression in Neurospora crassa. New insights into the $\mathrm{pH}$ signaling pathway. PLoS One 7:e44258.

Dementhon K, Iyer G and Glass NL (2006) VIB-1 is required for expression of genes necessary for programmed cell death in Neurospora crassa. Eukaryot Cell 5:2161-2173.

Dick CF, Dos-Santos AL and Meyer-Fernandes JR (2011) Inorganic phosphate as an important regulator of phosphatases. Enzyme Res 2011:103980.

Eisendle M, Oberegger H, Zadra I and Haas H (2003) The siderophore system is essential for viability of Aspergillus nidulans: functional analysis of two genes encoding 1-ornithine $N$ 5 -monooxygenase (sidA) and a non-ribosomal peptide synthetase ( $\operatorname{sid} C$ ). Mol Microbiol 49:359-375.

El-Ganiny AM, Sheoran I, Sanders DA and Kaminskyj SG (2010) Aspergillus nidulans UDP-glucose-4-epimerase UgeA has multiple roles in wall architecture, hyphal morphogenesis, and asexual development. Fungal Genet Biol 47:629-635.

Ferreira-Nozawa MS, Silveira HC, Ono CJ, Fachin AL, Rossi A and Martinez-Rossi NM (2006) The $\mathrm{pH}$ signaling transcription factor PacC mediates the growth of Trichophyton rubrum on human nail in vitro. Med Mycol 44:641-645.

Fortwendel JR (2015) Orchestration of morphogenesis in filamentous fungi: conserved roles for ras signaling networks. Fungal Biol Rev 29:54-62.

Gras DE, Silveira HC, Martinez-Rossi NM and Rossi A (2007) Identification of genes displaying differential expression in the nuc- 2 mutant strain of the mold Neurospora crassa grown under phosphate starvation. FEMS Microbiol Lett 269:196200.

Gras DE, Persinoti GF, Peres NT, Martinez-Rossi NM, Tahira AC, Reis EM, Prade RA and Rossi A (2013) Transcriptional profiling of Neurospora crassa $\Delta m a k-2$ reveals that mitogenactivated protein kinase MAK-2 participates in the phosphate signaling pathway. Fungal Genet Biol 60:140-149.

Han SW, Nahas E and Rossi A (1987) Regulation of synthesis and secretion of acid and alkaline phosphatases in Neurospora crassa. Curr Genet 11:521-527.

Hartman JL, Garvik B and Hartwell L (2001) Principles for the buffering of genetic variation. Science 291:1001-1004.

Hissen AH, Wan AN, Warwas ML, Pinto LJ and Moore MM (2005) The Aspergillus fumigatus siderophore biosynthetic gene sidA, encoding L-ornithine N5-oxygenase, is required for virulence. Infect Immun 73:5493-5503.

Kana-uchi A, Yamashiro CT, Tanabe S and Murayama T (1997) A ras homologue of Neurospora crassa regulates morphology. Mol Gen Genet 254:427-432.

Landraud P, Chuzeville S, Billon-Grande G, Poussereau N and Bruel C (2013) Adaptation to $\mathrm{pH}$ and role of $\mathrm{PacC}$ in the rice blast fungus Magnaporthe oryzae. PLoS One 8:e69236.

Li Y, Wang Z, Liu X, Song Z, Li R, Shao C and Yin Y (2016) Siderophore biosynthesis but not reductive iron assimilation is essential for the dimorphic fungus Nomuraea Rileyi conidiation, dimorphism transition, resistance to oxidative stress, pigmented microsclerotium formation, and virulence. Front Microbiol 7:931.

Maddi A, Bowman SM and Free SJ (2009) Trifluoromethanesulfonic acid-based proteomic analysis of cell wall and secreted proteins of the ascomycetous fungi Neurospora crassa and Candida albicans. Fungal Genet Biol 46:768-781. 
Mani R, St Onge RP, Hartman JL, Giaever G and Roth FP (2008) Defining genetic interaction. Proc Natl Acad Sci USA 105:3461-3466.

Martinez-Rossi NM, Peres NT and Rossi A (2017) Pathogenesis of dermatophytosis: Sensing the host tissue. Mycopathologia 182:215-227.

Martins MP, Gomes EV, Sanches PR, Pedersoli WR, MartinezRossi NM and Rossi A (2018) mus-52 disruption and metabolic regulation in Neurospora crassa: Transcriptional responses to extracellular phosphate availability. PLoS One 13:e0195871.

Martins MP, Martinez-Rossi NM, Sanches PR, Gomes EV, Bertolini MC, Pedersoli WR, Silva RN and Rossi A (2019) The $\mathrm{pH}$ signaling transcription factor PAC-3 regulates metabolic and developmental processes in pathogenic fungi. Front Microbiol 10:2076.

Melin P, Schnurer J and Wagner EG (2003) Characterization of phiA, a gene essential for phialide development in Aspergillus nidulans. Fungal Genet Biol 40:234-241.

Mendes NS, Trevisan GL, Silva Cruz AH, Santos RS, Peres NT, Martinez-Rossi NM and Rossi A (2012) Transcription of Nand O-linked mannosyltransferase genes is modulated by the pacC gene in the human dermatophyte Trichophyton rubrum. FEBS Open Bio 2:294-297.

Mendes NS, Silva PM, Silva-Rocha R, Martinez-Rossi NM and Rossi A (2016) Pre-mRNA splicing is modulated by antifungal drugs in the filamentous fungus Neurospora crassa. FEBS Open Bio 6:358-368.

Michan S, Lledias F and Hansberg W (2003) Asexual development is increased in Neurospora crassa cat-3-null mutant strains. Eukaryot Cell 2:798-808.

Morales Hernandez CE, Padilla Guerrero IE, Gonzalez Hernandez GA, Salazar Solis E and Torres Guzman JC (2010) Catalase overexpression reduces the germination time and increases the pathogenicity of the fungus Metarhizium anisopliae. Appl Microbiol Biotechnol 87:1033-1044.

Mouyna I, Fontaine T, Vai M, Monod M, Fonzi WA, Diaquin M, Popolo L, Hartland RP and Latge JP (2000) Glycosylphosphatidylinositol-anchored glucanosyltransferases play an active role in the biosynthesis of the fungal cell wall. J Biol Chem 275:14882-14889.

Nyc JF, Kadner RJ and Crocken BJ (1966) A repressible alkaline phosphatase in Neurospora crassa. J Biol Chem 241:14681472.

O'Meara TR, Xu W, Selvig KM, O'Meara MJ, Mitchell AP and Alspaugh JA (2014) The Cryptococcus neoformans Rim101 transcription factor directly regulates genes required for adaptation to the host. Mol Cell Biol 34:673-684.

Potapova TV (2014) Structural and functional organization of growing tips of Neurospora crassa hyphae. Biochemistry (Mosc) 79:593-607.

Ragni E, Calderon J, Fascio U, Sipiczki M, Fonzi WA and Popolo L (2011) Phrlp, a glycosylphosphatidylinsitol-anchored beta(1,3)-glucanosyltransferase critical for hyphal wall formation, localizes to the apical growth sites and septa in Candida albicans. Fungal Genet Biol 48:793-805.

Rascle C, Dieryckx C, Dupuy JW, Muszkieta L, Souibgui E, Droux M, Bruel C, Girard V and Poussereau N (2018) The pH regulator PacC: a host-dependent virulence factor in Botrytis cinerea. Environ Microbiol Rep 10:555-568.

Rerngsamran P, Murphy MB, Doyle SA and Ebbole DJ (2005) Fluffy, the major regulator of conidiation in Neurospora cras- $s a$, directly activates a developmentally regulated hydrophobin gene. Mol Microbiol 56:282-297.

Rodriguez-Kessler M, Baeza-Montanez L, Garcia-Pedrajas MD, Tapia-Moreno A, Gold S, Jimenez-Bremont JF and RuizHerrera $\mathbf{J}$ (2012) Isolation of UmRrm 75, a gene involved in dimorphism and virulence of Ustilago maydis. Microbiol Res 167:270-282.

Rossi A, Cruz AH, Santos RS, Silva PM, Silva EM, Mendes NS and Martinez-Rossi NM (2013) Ambient pH sensing in filamentous fungi: pitfalls in elucidating regulatory hierarchical signaling networks. IUBMB Life 65:930-935.

Silverman-Gavrila LB and Lew RR (2001) Regulation of the tiphigh $\left[\mathrm{Ca}^{2+}\right]$ gradient in growing hyphae of the fungus Neurospora crassa. Eur J Cell Biol 80:379-390.

Simaan H, Lev S and Horwitz BA (2019) Oxidant-sensing pathways in the responses of fungal pathogens to chemical stress signals. Front Microbiol 10:567.

Singh V, Satheesh SV, Raghavendra ML and Sadhale PP (2007) The key enzyme in galactose metabolism, UDP-galactose4-epimerase, affects cell-wall integrity and morphology in Candida albicans even in the absence of galactose. Fungal Genet Biol 44:563-574.

Sun X, Yu L, Lan N, Wei S, Yu Y, Zhang H, Zhang X and Li S (2012) Analysis of the role of transcription factor VAD-5 in conidiation of Neurospora crassa. Fungal Genet Biol 49:379-387.

Suzuki S, Sarikaya Bayram O, Bayram O and Braus GH (2013) conF and conJ contribute to conidia germination and stress response in the filamentous fungus Aspergillus nidulans. Fungal Genet Biol 56:42-53.

Tilburn J, Sarkar S, Widdick DA, Espeso EA, Orejas M, Mungroo J, Penalva MA and Arst Jr HN (1995) The Aspergillus PacC zinc finger transcription factor mediates regulation of both acid- and alkaline-expressed genes by ambient $\mathrm{pH}$. EMBO J 14:779-790.

Trevisan GL, Oliveira EH, Peres NT, Cruz AH, Martinez-Rossi NM and Rossi A (2011) Transcription of Aspergillus nidulans pacC is modulated by alternative RNA splicing of palB. FEBS Lett 585:3442-3445.

Vicent I, Navarro A, Mulet JM, Sharma S and Serrano R (2015) Uptake of inorganic phosphate is a limiting factor for Saccharomyces cerevisiae during growth at low temperatures. FEMS Yeast Res 15: fov008.

Virgilio S, Cupertino FB, Bernardes NE, Freitas FZ, Takeda AA, Fontes MR and Bertolini MC (2016) Molecular components of the Neurospora crassa $\mathrm{pH}$ signaling pathway and their regulation by $\mathrm{pH}$ and the PAC-3 transcription factor. PLoS One 11:e0161659.

Vogel HJ (1956) A convenient growth medium for Neurospora crassa (medium N). Microb Genet Bull 13:42-43.

Zelter A, Bencina M, Bowman BJ, Yarden O and Read ND (2004) A comparative genomic analysis of the calcium signaling machinery in Neurospora crassa, Magnaporthe grisea, and Saccharomyces cerevisiae. Fungal Genet Biol 41:827-841.

Zhang L, Feng D, Fang W, Ouyang H, Luo Y, Du T and Jin C (2009) Comparative proteomic analysis of an Aspergillus fumigatus mutant deficient in glucosidase I (AfCwh41). Microbiology 155:2157-2167.

\section{Internet Resources}

Carlson M (2018) GO.db: A set of annotation maps describing the entire Gene Ontology. R Package Version 3.7.0.

Ensembl Fungi, https://fungi.ensembl.org/index.html. 


\section{Supplementary Material}

The following online material is available for this article:
Table S1 - Genes of $N$. crassa modulated in response to mutant strain $\triangle p a c C$ (test) compared with the control strain (Amus-52) in medium containing low-Pi or high-Pi.

Associate Editor: Célia Maria de Almeida Soares

License information: This is an open-access article distributed under the terms of the Creative Commons Attribution License (type CC-BY), which permits unrestricted use, distribution and reproduction in any medium, provided the original article is properly cited. 